Supporting information for:

\title{
A ReaxFF reactive force field for disulfide mechanochemistry, fitted to multireference ab-initio data
}

Julian Müller, and Bernd Hartke*

Institute for Physical Chemistry,

Christian-Albrechts-University,

Olshausenstr. 40,

24098 Kiel, GERMANY 


\section{Setting up parameter boundaries}

To illustrate our force-field parameter optimization procedures with a more concrete example, we describe here how the intervals were found within which the force-field parameters were allowed to vary. As we already mentioned in the main text, this is one of the crucial tasks in a successful reparameterization.

There are a few ways to generate an initial guess for the params file, which contains the optimizable parameters and their boundaries (see below). One option is to simply allow for an interval of reasonable width around a parameter value known from a related force field. Another way would be to look at the existing parameterizations and use the lowest and highest values found, plus a tolerance. However, such a first guess seldom suffices to yield reliable and satisfying results within reasonable computing time. The search space might be too big to converge to useful regions or too small to include the desired solutions. Furthermore the search space could include regions that yield low deviation solutions but will result in severe instabilities when the force field is applied to MD calculations. Thus, refinement of the parameter boundaries is mandatory. In the following, a few methods for refinement that were used in this work are outlined.

The first, most obvious possibility to refine the parameter set and reduce instabilities is to look for parameters that have a direct physical interpretation. Parameters of this kind are, for example, equilibrium distances, van der Waals radii or dissociation energies. Once they are identified, it is relatively easy to set narrow boundaries around the assumed ideal value, which will reduce the size of the search space significantly. However, due to the intricacies of the REAXFF formalism, such direct physical links often are weaker than expected. For example, $\sigma$-equilibrium bond length parameters are smaller than the expected bond length would suggest, while $D_{e}$ parameters are significantly bigger than the actual dissociation energies.

Another option is to use test optimizations with rather big pools and observe how the parameter values are distributed depending on the respective pool ranks. When performing such test calculations, the niching must be set up first to meet the demands of these benchmarks. When no niching is used, the solutions will collapse to one single vector and no information about the search space can be retrieved from the pool. When the niching is to restrictive, the individuals are forced to spread over the whole search space and no localization or preference of the parameters can be observed. Figures 1-3 show different scenarios how the resulting individuals can behave after an optimization and how to act on them.

As a concrete example, we have picked parameter 2-4-4 shown in figure 1 . This parameter controls the van der Waals radius of the sulfur atom. Looking at the parameterizations available in the LAMMPS potential library, an average value of about $2 \AA$ can be found for this parameter.

Hence, as an initial guess for the boundaries, a range of 1.5-2.5 was chosen. Then test optimizations were performed. Two conclusions were drawn from these calculations. First, a localization of the parameter around the value 1.9 could be observed. Second, the resulting force field showed instabilities when the parameter was above 2.2. For the refinement step, the boundaries were therefore set to 1.5-2.2, which yielded the scatter plot shown in figure 1. Obviously, at the "better end" (low rank numbers) of the pool, the individuals consistently narrow down towards the smaller range 1.8-2.0, while at the other end of the pool the whole range of 1.5-2.2 is explored. This indicates that the 
range $1.5-2.2$ presumably is reasonable.

The interested reader might have noticed that the actual boundaries for the parameter shown in figure 1 in the params file are 1.7-2.0. This is due to the fact that the parameter distributions are taken from an intermediate step along the road from the initial guess for parameters and reference set to the final setup used for the optimization. The steps described above were repeated many times, and shifting boundaries or adding parameters also shift the sweet spots in the distribution of parameters that are plotted.

Figures 2 and 3 illustrate other specific cases where obvious further parameter range refinement is necessary.

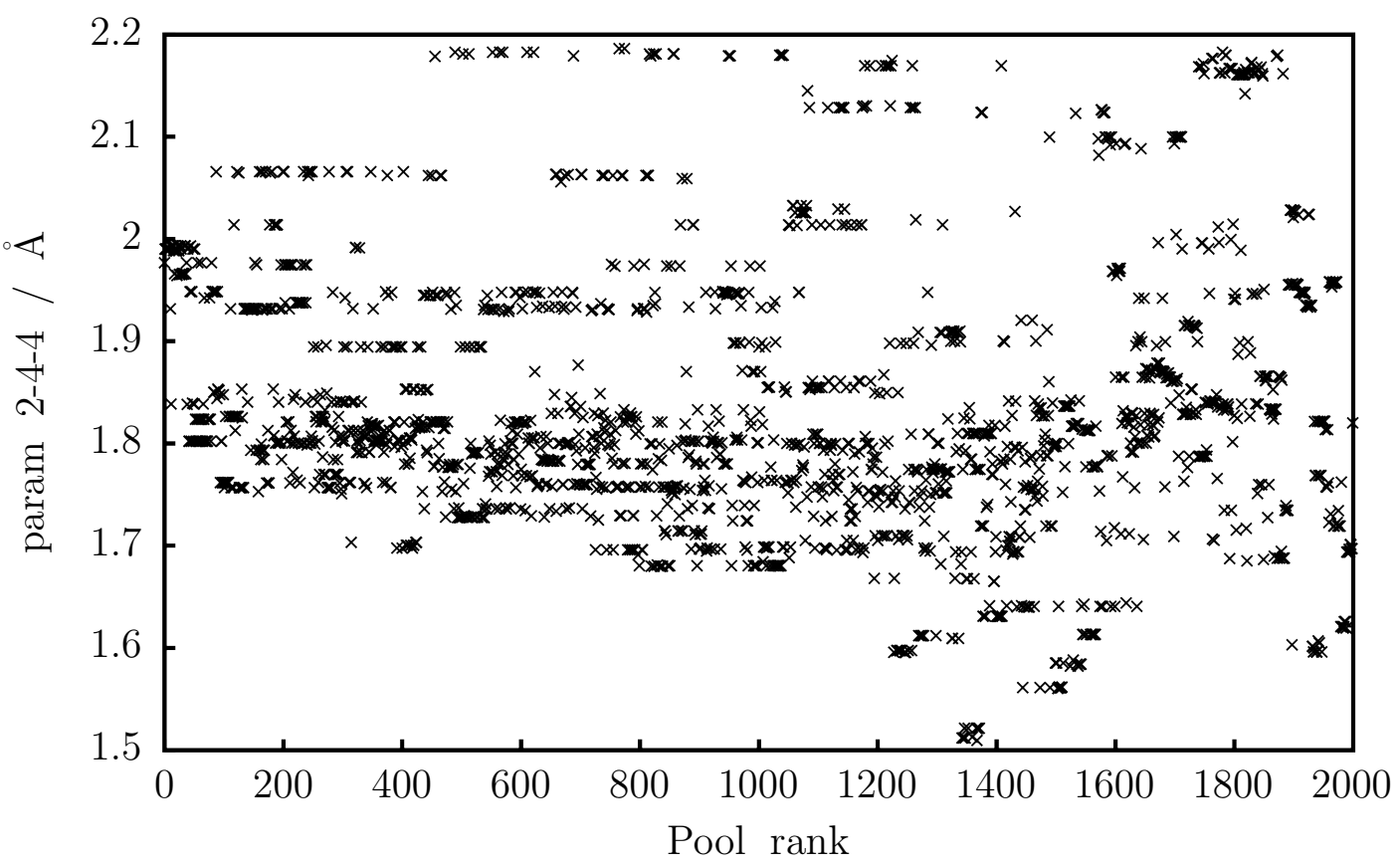

Fig. 1: Good spreading of the individuals over the available search space. No need for any refinement. 


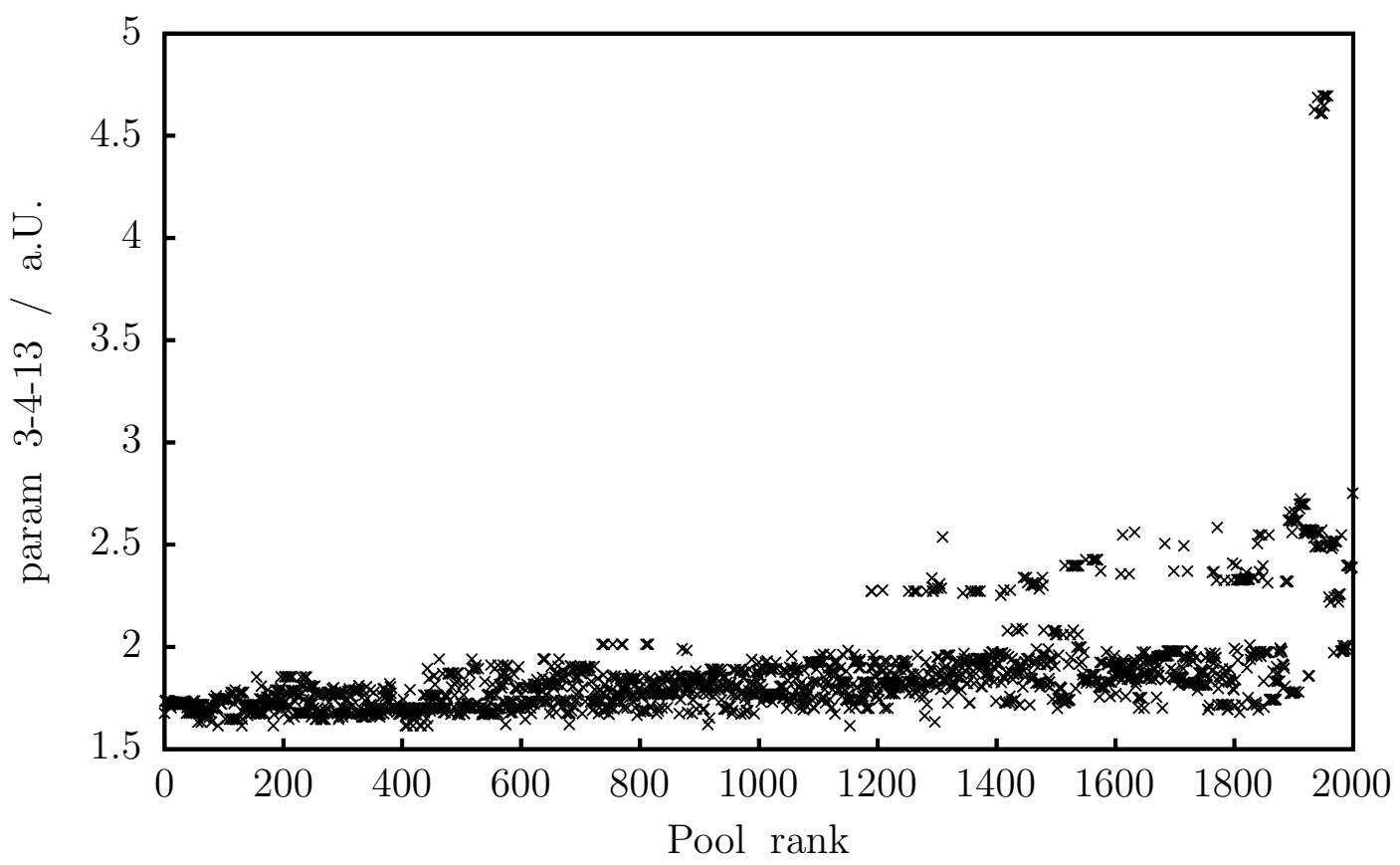

Fig. 2: Strong localization of the individuals towards a narrow region of the search space. Refinement of the params file will be to reduce the range of this parameter.

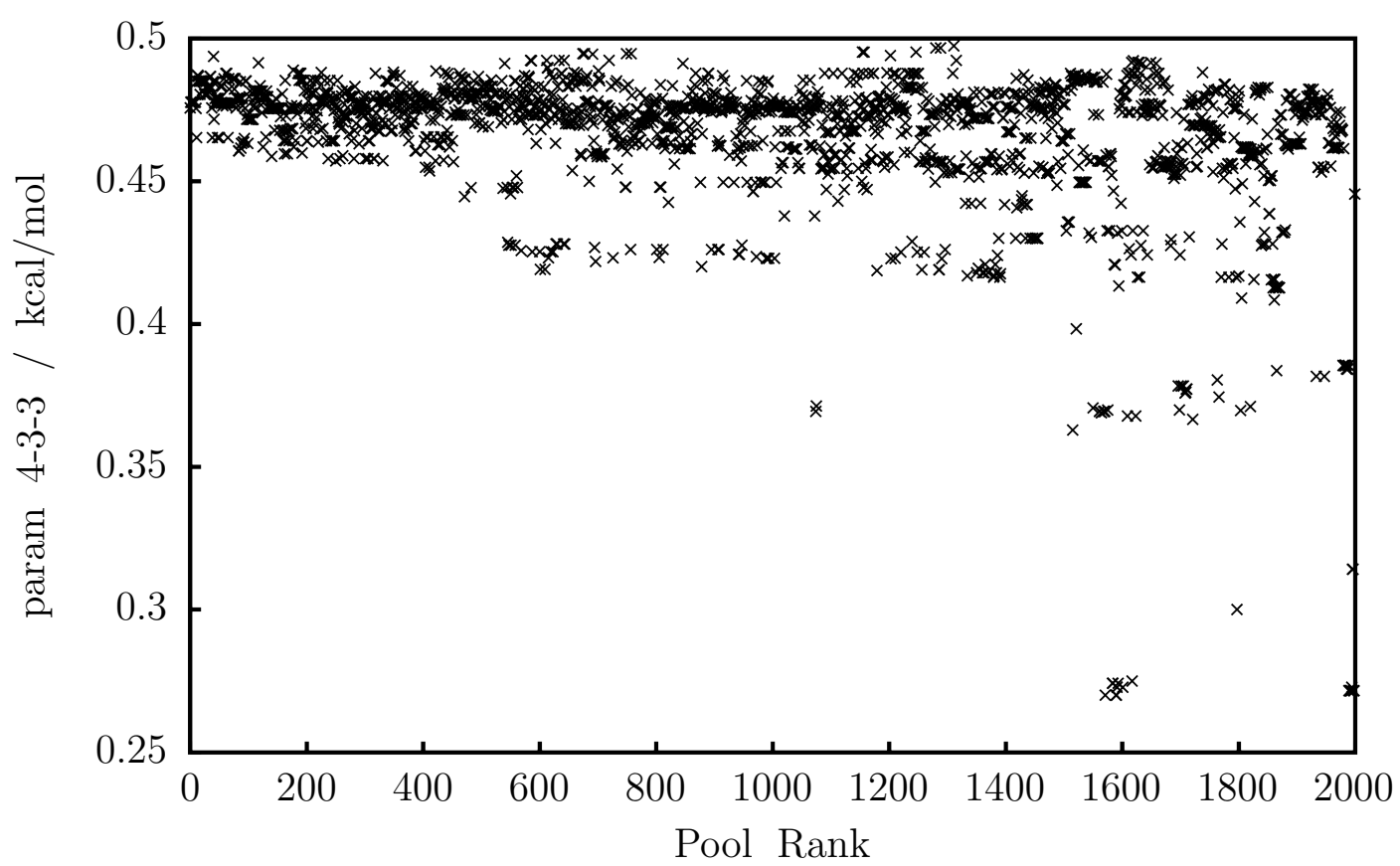

Fig. 3: Localization towards the upper boundary of the searchspace in this parameter (0.5). To refine the params file the boundaries should be narrowed a bit and shifted towards higher values.

\section{Supporting files}

With the supporting information two sets of files are provided, one contains the reference set used in the optimization, the other is the validation set used to determine the early stopping. 
A more detailed description of the input files may be found in the REAxFF user manual [1], including the file formats and the m-n-k nomenclature for ReaxFF parameters which we have employed in the above paragraph and in Figs.1-3.

\section{1 ffield}

The ffield file provided with the supporting info contains the optimized parameters of the Mue2016 force field, as obtained in the global optimization described in the main text.

\section{2 params}

The params file holds information about the parameters that are to be optimized and the boundaries of these parameters in the search space. Although the input format is compatible with the original parameter optimization with SOPPE as used by van Duin, the step size for SOPPE is set to a dafault value of 0.0050 for all parameters. This value is simply not needed for an optimization with EAs, and omitting it may cause problems when using this reference set with SOPPE.

\subsection{1 trainset.in}

The trainset files contain all the reference values and the weighting factors needed to construct the objective function for the optimization. Relative energies, geometric properties and gradients are given here, and referenced to structures in the geo file. The relative energies are given in $\mathrm{kcal} / \mathrm{mol}$, bond lengths, angles and dihedrals in $\AA$ and degree, respectively, and gradients in Hartree/Bohr.

\section{3 geo}

The geo files contain all molecular input structures used in the optimization and the early stopping, in the biograf 200 format used by REAxFF.

Table 1: Overview of the molecular structures used in the reference set. Actual structures are given as biograf 200 structures in the respective geo file.

Structure identifier Full name Structure identifier Full name

\begin{tabular}{|c|c|c|c|}
\hline hsshGeo & Hydrogen disulfide & h2sGeo & $\begin{array}{c}\text { Hydrogen sulfide } \\
\mathrm{H}^{-} \mathrm{S}_{\longrightarrow}{ }_{\mathrm{H}}\end{array}$ \\
\hline mdtBase & Methanedithiol & dmteBase & Dimethyl sulfide \\
\hline
\end{tabular}


$S_{8}$ allotrope of sulfur

dmds Dimethyl disulfide s8<smiles>S1SSSSSSS1</smiles>

2,2'-Dithiophenol

Diphenyl disulfide

dpds<smiles>CSc1ccccc1</smiles><smiles>Sc1ccccc1</smiles>

dpods<smiles>Cc1ccccc1O</smiles><smiles>Oc1ccccc1SS</smiles>

Table 2: Overview of the molecular structures used in the validation set. Actual structures are given as biograf 200 structures in the respective geo file.

dpds

Diphenyl disulfide<smiles>Sc1ccccc1</smiles>

S<smiles>Ic1ccccc1</smiles>

Dimethyl disulfide

dmds<smiles>CSSC</smiles>

edt

dpods

\section{2,2'-Dithiophenol}<smiles>Cc1ccccc1O</smiles><smiles>Oc1ccccc1SS</smiles>

1,2-Ethanedithiol<smiles>SCCS</smiles>

Toluene

tou<smiles>Cc1ccccc1</smiles> 
pho<smiles>Oc1ccccc1</smiles>

Thiobenzophenone

dpt<smiles>S=C(c1ccccc1)c1ccccc1</smiles>

Ethylphenylether

epe<smiles>CCOc1ccccc1</smiles>

Cyclopentathion

cpt<smiles>S=C1CCCC1</smiles>

Pentathiolacton

cptl<smiles>S=C1CCCS1</smiles>

Thiophene

dht<smiles>c1ccsc1</smiles>

Hygrogen disulfide

hssh

$$
\mathrm{H}^{-}{ }^{-} \mathrm{S}^{-} \mathrm{H}^{-}
$$

Ethane(dithionic) acid

taa<smiles>CC(=S)S</smiles>

tpho

Thiophenol<smiles>Sc1ccccc1</smiles>

Thioanisol

tani<smiles>CSc1ccccc1</smiles>

Thian

tch<smiles>C1CCSCC1</smiles>

2-Cyclopentene-1-Thion

cpet<smiles>S=C1C=CCC1</smiles>

Cyclohexathion

cht<smiles>S=C1CCCCC1</smiles>

Tetrahydrothiophene

thp<smiles>C1CCSC1</smiles>

Hydrogen sulfide<smiles>S</smiles>

Methyldithioacetate

mte<smiles>CSC(C)=S</smiles> 


dmt ett

The structures in table 1 are the MP2-optimized equilibrium structures used in the geometry part of the reference set. The second table 2 shows the MP2-optimized structures used in the validation set.

The derived structure names in the geo files indicate that those are structures along scan coordinates used in the optimization. For example, dmds-SS3.5 denotes a structure for dimethyl disulfide along a rigid scan, with the SS distance set to $3.5 \AA$. Another example would be dmds-CSSCr225, which indicates that the CSSC coordinate was held fixed at 225 degrees and the remaining atoms were allowed to relax locally.

All other derived names (e.g. dmds-SS3.5) denote the coordinate and the value of that coordinate along a rigid scan. These structures are used for the gradient and relative energy calculations.

\section{$2.4 \operatorname{grads} / *$}

The grads directory contains all gradients used in the optimization, in the xyz format. Since it it not always desired to have the gradients of every atom in a structure contributing to the objective value, the format of the gradient is the atom number of the atom in the structure as found in the geo file, followed by the three cartesian gradient contributions in atomic units.

\section{References}

[1] https://www.scm.com/wp-content/uploads/ReaxFF-users-manual-2002.pdf 